\title{
Implementation of Highly-Flowable Strain Hardening Fiber Reinforced Concrete in New RC Beam-Column Joints
}

\author{
Wen-Cheng Liao ${ }^{1 *}$, Wei-Ru $\mathrm{Su}^{2}$ \\ ${ }^{1}$ Associate Professor, Department of Civil Engineering, National Taiwan University, Taipei, Taiwan \\ ${ }^{2}$ Graduate Student, Department of Civil Engineering, National Taiwan University, Taipei, Taiwan
}

\begin{abstract}
The purpose of New RC project was aimed to reduce the member sections and increase the available space of high rise buildings by using high strength concrete ( $\mathrm{f}_{\mathrm{c}}>70 \mathrm{MPa}$ ) and high strength rebars ( $\left.f_{y}>685 \mathrm{MPa}\right)$. Material consumptions and member section sizes can be further reduced owing to the upgrade of strength. However, the nature of brittleness of high strength may also cause early cover spalling and other ductility issues. Addition of steel fibers is an alternative as transverse reinforcement. Highly flowable strain hardening fiber reinforced concrete (HF-SHFRC) has excellent workability in the fresh state and exhibits the strain-hardening and multiple cracking characteristics of high performance fiber reinforced cementitious composites (HPFRCC) in their hardened state. The objective of this study is to investigate the feasibility of implementing HF-SHFRC in New RC building systems, particularly for beam-column joints as an alternative of transverse reinforcements. Four full-scale exterior beam-column joints, including two specimens with intensive transverse reinforcements and two specimens made of HF-SHFRC without any stirrup, are tested. Test results show that the HF-SHFRC specimens perform as well as specimens with intensive transverse reinforcements regarding failure mode, ductility, energy dissipation and crack width control. Integration of New RC building systems and HF-SHFRC can assuring construction qualities and further diminish labor work and give infrastructure longer service life, and eventually lower the life-cycle cost.
\end{abstract}

\section{Introduction}

The purpose of New RC project was aimed to reduce the member sections and increase the available space of high rise buildings by using high strength concrete ( $\mathrm{f}^{\prime}{ }_{\mathrm{c}}>70$ $\mathrm{MPa})$ and high strength rebars $\left(\mathrm{f}_{\mathrm{y}}>685 \mathrm{MPa}\right)$. Material consumptions and member sections can be further reduced owing to the upgrade of strength. However, the nature of brittleness of high strength may also cause early cover spalling and other ductility issues. Addition of steel fibers is an alternative as transverse reinforcement. Highly flowable strain hardening fiber reinforced concrete (HF-SHFRC) has excellent workability in the fresh state and exhibits the strainhardening and multiple cracking characteristics of high performance fiber reinforced cementitious composites (HPFRCC) in their hardened state.

A beam-column joint takes an important role in the force transferring mechanism in a special moment resisting frame. Thus, severe damage within a joint panel may cause deterioration in overall seismic performance of the frame. As a joint is subjected to high shear strength, code recommendations suggest arranging closely hoop transverse reinforcement to prevent joins from shear failure, which leads congested joints and results in difficult construction works. With high tensile strength and good toughness behavior, applying HF-
SHFRC in joint panels is expected to reduce the amount of transverse reinforcement. The objective of this study is to investigate the feasibility of implementing HFSHFRC in New RC building systems, such as beamcolumn joints and base columns as an alternative of transverse reinforcements. Test results show that the HFSHFRC specimens perform as well as specimens with intensive transverse reinforcements regarding failure mode, ductility, energy dissipation and crack width control.

\section{Experimental programs}

Four full-scale exterior beam-column joints, including two specimens with intensive transverse reinforcements and two specimens made of HF-SHFRC without any stirrup, are tested.

\subsection{Materials}

The cementitious materials used in this study were ASTM Type I Portland cement; ground granulated blast furnace slag and class $\mathrm{C}$ fly ash. The coarse aggregate had a maximum size of $9.5 \mathrm{~mm}$ and consisted of solid crushed limestone from a local source, with a density of about $\quad 2.70 \quad \mathrm{~g} / \mathrm{cm}^{3}$. A polycarboxylate-based

\footnotetext{
* Corresponding author: wcliao@ntu.edu.tw
} 
superplasticizer was used in the mixture. Hooked steel fiber with circular cross-section was used, with normal tensile strength of $2300 \mathrm{MPa}$ and aspect ratio of 79 . The concrete compressive strengths were determined in accordance with ASTM C-39 standard compressive tests on at least six $100 \times 200 \mathrm{~mm}$ cylinders. The concrete specified compressive strengths were 70 and $100 \mathrm{MPa}$. The slump flow of HF-SHFRC used in this study is $580 \mathrm{~mm}$ so that the workability is not the issue during fabrication. Two different sizes of reinforcing bars were used: D25 $\left(5.07 \mathrm{~cm}^{2}\right)$ with yield stress of $685 \mathrm{MPa}$ for longitudinal reinforcement, and D13 $\left(1.27 \mathrm{~cm}^{2}\right)$ with yield stress of $785 \mathrm{MPa}$ for transverse reinforcement. The baseline tensile test for a single control reinforcing bar to determine yield strength, ultimate strength and bar strain at yield stress in accordance with ASTM E8/E8M and ASTM A370.

\subsection{Specimen design}

The seismic performances of exterior beam-column joints are assessed by applying a double curvature, cyclic lateral loading experiment. The columns had a clear height of $2800 \mathrm{~mm}$ with a square cross-section of $600 \times 600 \mathrm{~mm}$. Beam sectional size is $400 \times 700 \mathrm{~mm}$ and the net length is $3500 \mathrm{~mm}$. The four beam-column joints are labeled as LAMV, HAMV, LAMV_SF and HAMV_SF, respectively. LA represents as low axial load which is equal to $0.1 \mathrm{Ag} / \mathrm{f}_{\mathrm{c}}$; HA represents as high axial load which is equal to $0.45 \mathrm{Ag} / \mathrm{f}^{\prime}$. MV represents medium shear ratio which is defined as the ratio of shear demand $\mathrm{V}_{\mathrm{jh}, \mathrm{u}}$ and shear capacity $\mathrm{V}_{\mathrm{n}}$ of joints, which is 0.7 in this experimental program, where $\mathrm{V}_{\mathrm{jh}, \mathrm{u}}$ is the total horizontal joint shear force and $\mathrm{V}_{\mathrm{n}}$ is the nominal shear strength of joint.

LAMV and HAMV are considered as control group (Chao [3]), and LAMV_SF and HAMV_SF are the experimental group. All these specimens have the identical flexural strength in column and beam section since their reinforcement layouts of beam and column sections are the same. The joints of LAMV and HAMV consist of dense transverse reinforcement to comply with ACI 318-11, while no transverse reinforcement is arranged in joints by using HF-SHFRC instead in LAMV_SF and HAMV_SF. The design parameters are listed in Table 1.

\subsection{Test setup}

The test of the specimens performed in National Center for Research on Earthquake Engineering (NCREE) using Multi-Axial Resting System (MATS) that built in 2007. The MATS conducted double-curvature cyclic deformation. The maximum axial and lateral load that can be applied by MATS is $60 \mathrm{MN}$ and $7 \mathrm{MN}$, respectively. The lateral force was set from the hydraulic actuator that placed in the bottom as shown in Fig. 1(a) and the loading protocol is shown in Fig. 1(b). For each cycle of each loading phase, once target drift was reached, loading was temporarily stopped and the crack patterns were marked and recorded. The experiment was continued until a significant loss in axial loading capacity of column was observed. Significant loss in axial loading capacity was interpreted using the following guidelines: rapid reduction in axial loading capacity; or sudden MATS loading stroke greater than or equal to $20 \mathrm{~mm}$. Experiments in this study were conducted under high axial forces, resultantly the P- $\Delta$ effect was noticeable.

Table 1 Design parameters

\begin{tabular}{|c|c|c|c|c|c|}
\hline $\begin{array}{c}\text { Specimen } \\
\text { ID } \\
\text { Section } \\
\end{array}$ & $\begin{array}{l}\frac{A_{5 h}}{B b_{2}}(\%) \\
V_{f}(\%)\end{array}$ & $\begin{array}{c}\mathrm{f}^{\prime}{ }_{c} \\
(\mathrm{MPa})\end{array}$ & $\begin{array}{c}f_{y} \\
(\mathrm{MPa})\end{array}$ & $\begin{array}{c}f_{y t} \\
(\mathrm{MPa})\end{array}$ & $\frac{\mathrm{N}_{\mathrm{u}}}{\mathrm{A}_{\mathrm{g}^{\mathrm{x}}} \mathrm{f}_{\mathrm{c}}^{\mathrm{s}}}$ \\
\hline 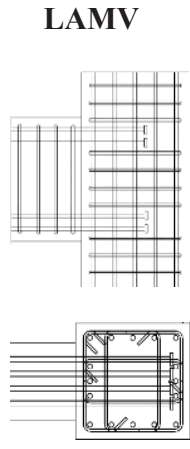 & $\begin{array}{c}1.0 \\
0\end{array}$ & 70 & $\begin{array}{c}685 \\
\text { (D25) }\end{array}$ & $\begin{array}{c}785 \\
\text { (D13) }\end{array}$ & 0.1 \\
\hline 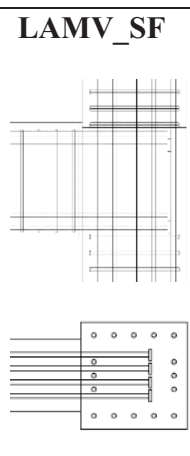 & $\begin{array}{c}0 \\
1.5\end{array}$ & 70 & $\begin{array}{c}685 \\
\text { (D25) }\end{array}$ & - & 0.1 \\
\hline 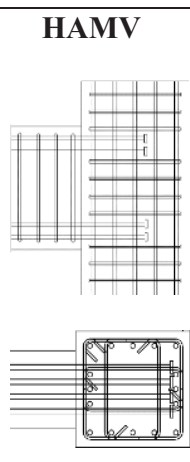 & $\begin{array}{c}1.0 \\
0\end{array}$ & 70 & $\begin{array}{c}685 \\
(\mathrm{D} 25)\end{array}$ & $\begin{array}{c}785 \\
\text { (D13) }\end{array}$ & 0.45 \\
\hline 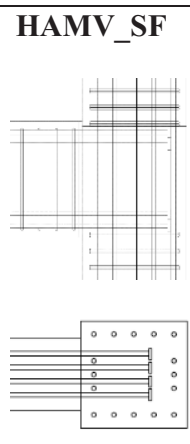 & 1.5 & 70 & $\begin{array}{c}685 \\
\text { (D25) }\end{array}$ & - & 0.45 \\
\hline
\end{tabular}




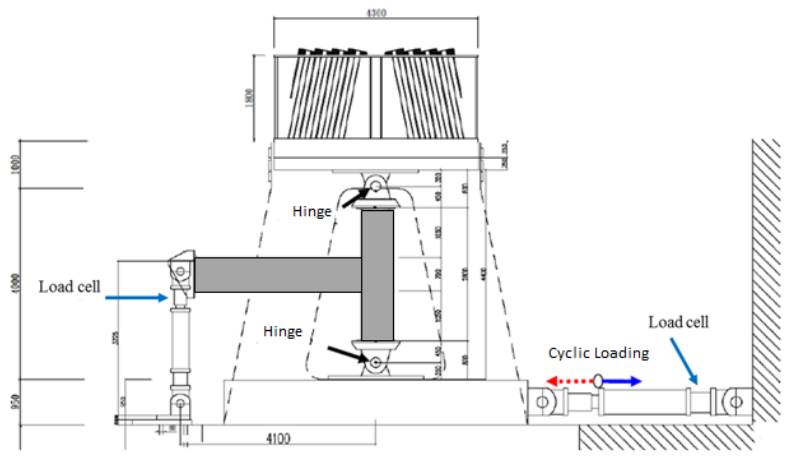

(a)

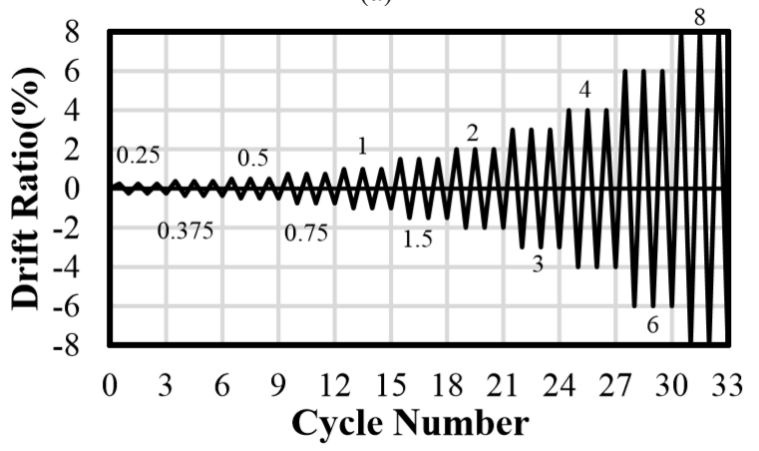

(b)

Fig. 1 (a) Test setup with MATS; (b) Loading protocol

\section{Test results}

\subsection{LAMV and LAMV_SF}

Figure 2 (a) shows the hysteresis loops of LAMV and LAMV_SF, which are modified with P-Delta effect. Their hysteresis loop envelopes are also shown in figure 2 (b). The apparent yielding plateau can be found in both LAMV and LAMV_SF, which stand for the B type failure mode. Their hysteresis loops and envelopes are almost identical since the yielding and failure of beam governs the cyclic behaviors. It is worth mentioning that there is no transverse reinforcement in the joint of LAMV SF.

Their hysteresis loops present a linear status before the story drift ratio reaches $0.29 \%$; as the story drift ratio exceeds $0.29 \%$, lateral force declines slowly along with the increase of the story drift ratio. The lateral force declines obviously till the second and third cycle of the $8 \%$ drift ratio loop. The maximum lateral forces of LAMV and LAMV SF are $487 \mathrm{kN}$ and $488 \mathrm{kN}$ as reaches $5.38 \%$ and $8 \%$ respectively. While the experiment progress to the third cycle of $6 \%$, the longitudinal steel bar in the beam occurred local buckling, and failed in the plastic zone as $8 \%$. The experiment ended up in the second cycle of $8 \%$.

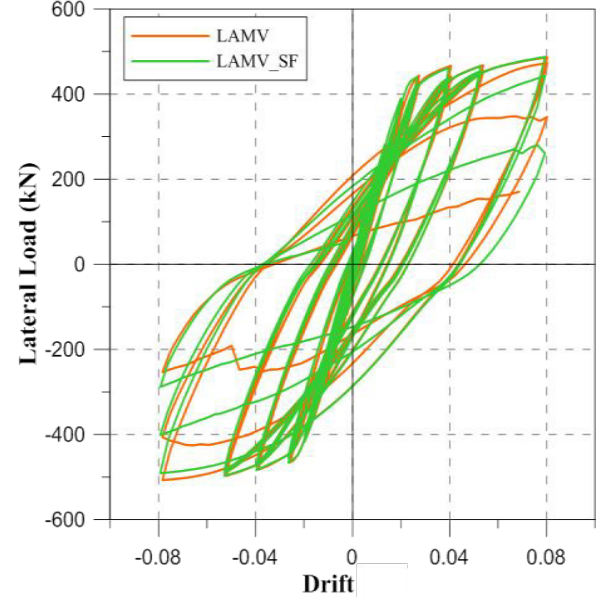

(a)

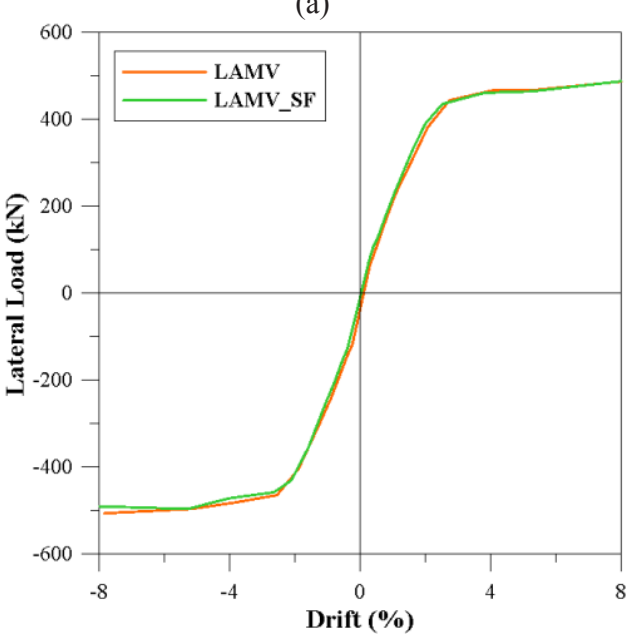

(b)

Fig. 2 (a) Hysteresis loops; (b) hysteresis loop envelopes of LAMV and LAMV SF

\subsection{HAMV and HAMV_SF}

Figure 3(a) illustrates the hysteresis loops of HAMV and HAMV SF, which are modified with P-Delta effect as well. Their hysteresis loop envelopes are compared in figure $3(\mathrm{~b})$. It is noted that the inversion of the hysteresis loop can be observed before modified with P-Delta effect due to additional lateral force caused by high axial load. Thus, the lateral force of MATS needs to be reversed to get the expected displacement. It is found that there is also a clear yield plateau in HAMV with B type failure mode. The specimen performs as a linear status before the story drift ratio reaches $0.27 \%$ and its maximum lateral forces are $478 \mathrm{kN}$ as reaches $\pm 7.93 \%$.

Almost identical cyclic behavior of HAMV_SF can be observed before the second cycle of $4 \%$ since it suddenly fails. From the photo at specimen failed and the hysteresis loop (Fig. 3(a)), it can be conjectured that the failure of HAMV_SF caused by inadequacy of confinement, rather than insufficiency of shear capacity as BJ type failure. Even though HAMV_SF failed at $4.0 \%$ drift ratio, its performance is still qualified as acceptable joint in terms of stiffness, strength and energy dissipation required by ACI 374 [2]. 


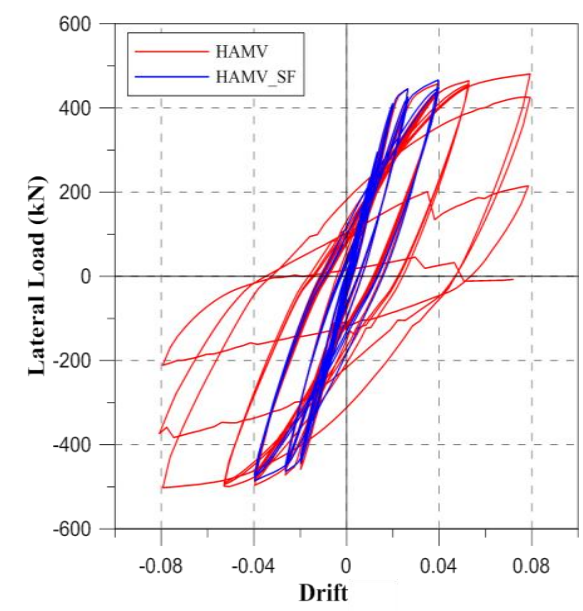

(a)

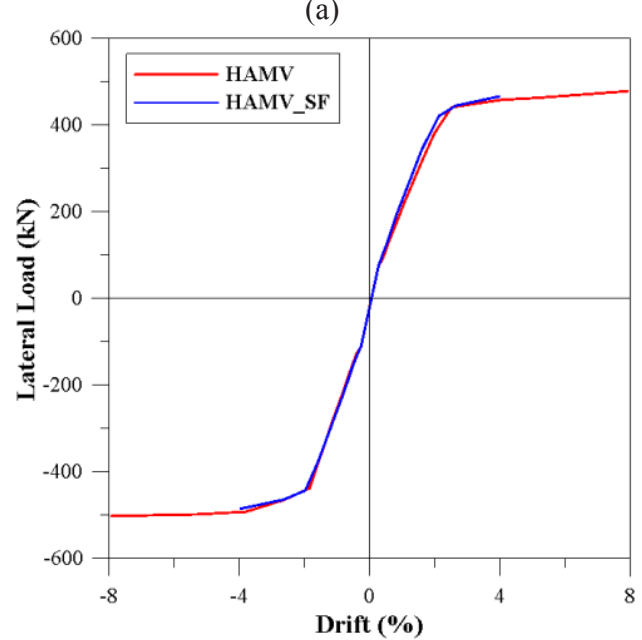

(b)

Fig. 3 (a) Hysteresis loops; (b) Hysteresis loop envelopes of HAMV and HAMV_SF

\section{Conclusions}

This study presents lateral cyclic loading tests on HFSHFRC beam-column joints to examine its adequacy to substitute transverse reinforcement in the joint region under different axial loading levels. LAMV_SF and LAMV have similar or even better cyclic behaviors, whereas HAMV_SF does not perform as well as HAMV on cracking width control, this implies HF-SHFRC can fully replace transverse reinforcement under low axial load. Proper transverse reinforcement should be applied for confinement purpose in the case of high axial load even though shear capacity of HF-SHFRC is sufficient. HF-SHFRC specimens perform as well as specimens with intensive transverse reinforcements regarding failure mode, ductility, energy dissipation and crack width control. Application of HF-SHFRC to New RC building systems can assuring construction qualities and further diminish labor work and give infrastructure longer service life, and eventually lower the life-cycle cost.

\section{References}

1. ACI Committee 318, Building Code Requirements for Structural Concrete (ACI 318-14), American Concrete Institute, (2014)

2. ACI Committee 374, Acceptance Criteria for Moment Frame Based on Structural Testing and Commentary, American Concrete Institute, (2005)

3. Chao, W.-F., Seismic Behavior of Headed-Bars in High-Strength RC Exterior Beam-Column Joints with P-Delta Effect, Master Thesis, National Taiwan University of Science and Technology, Taipei, Taiwan, (2016) 